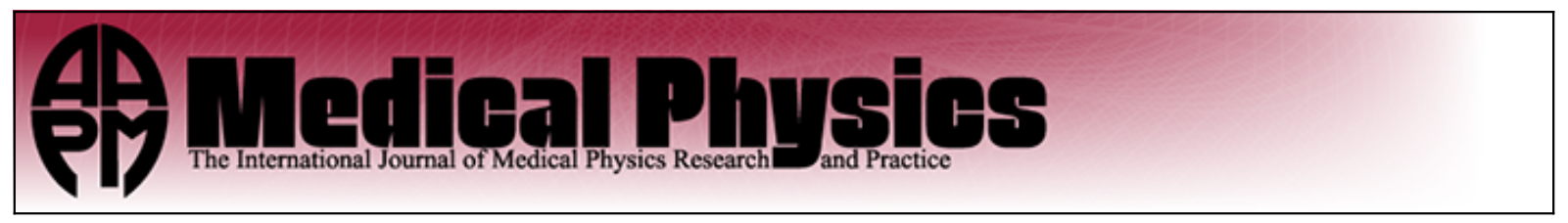

\title{
Temperature dependence of APD-based PET scanners
}

Vincent Keereman, Roel Van Holen, Stefaan Vandenberghe, and Christian Vanhove

Citation: Medical Physics 40, 092506 (2013); doi: 10.1118/1.4819823

View online: http://dx.doi.org/10.1118/1.4819823

View Table of Contents: http://scitation.aip.org/content/aapm/journal/medphys/40/9?ver=pdfcov

Published by the American Association of Physicists in Medicine

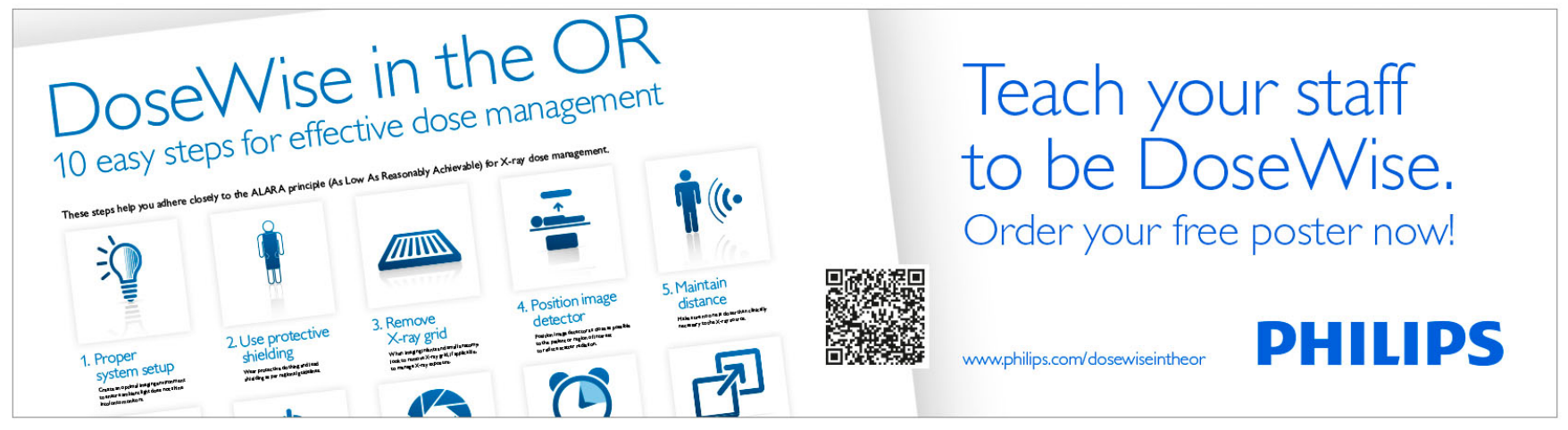




\title{
Temperature dependence of APD-based PET scanners
}

\author{
Vincent Keereman, ${ }^{a}$ Roel Van Holen, Stefaan Vandenberghe, and Christian Vanhove \\ MEDISIP, Department of Electronics and Information Systems, Ghent University-iMinds-IBiTech, \\ De Pintelaan 185, B-9000 Ghent, Belgium
}

(Received 31 January 2013; revised 6 August 2013; accepted for publication 10 August 2013; published 30 August 2013)

Purpose: Solid state detectors such as avalanche photodiodes (APDs) are increasingly being used in PET detectors. One of the disadvantages of APDs is the strong decrease of their gain factor with increasing ambient temperature. The light yield of most scintillation crystals also decreases when ambient temperature is increased. Both effects lead to considerable temperature dependence of the performance of APD-based PET scanners. In this paper, the authors propose a model for this dependence and the performance of the LabPET8 APD-based small animal PET scanner is evaluated at different temperatures.

Methods: The model proposes that the effect of increasing temperature on the energy histogram of an APD-based PET scanner is a compression of the histogram along the energy axis. The energy histogram of the LabPET system was acquired at $21^{\circ} \mathrm{C}$ and $25^{\circ} \mathrm{C}$ to verify the validity of this model. Using the proposed model, the effect of temperature on system sensitivity was simulated for different detector temperature coefficients and temperatures. Subsequently, the effect of short term and long term temperature changes on the peak sensitivity of the LabPET system was measured. The axial sensitivity profile was measured at $21^{\circ} \mathrm{C}$ and $24^{\circ} \mathrm{C}$ following the NEMA NU 4-2008 standard. System spatial resolution was also evaluated. Furthermore, scatter fraction, count losses and random coincidences were evaluated at different temperatures. Image quality was also investigated.

Results: As predicted by the model, the photopeak energy at $25^{\circ} \mathrm{C}$ is lower than at $21^{\circ} \mathrm{C}$ with a shift of approximately $6 \%$ per ${ }^{\circ} \mathrm{C}$. Simulations showed that this results in an approximately linear decrease of sensitivity when temperature is increased from $21^{\circ} \mathrm{C}$ to $24^{\circ} \mathrm{C}$ and energy thresholds are constant. Experimental evaluation of the peak sensitivity at different temperatures showed a strong linear correlation for short term $\left(2.32 \mathrm{kcps} / \mathrm{MBq} /{ }^{\circ} \mathrm{C}=12 \% /{ }^{\circ} \mathrm{C}, \mathrm{R}=-0.95\right)$ and long term $\left(1.92 \mathrm{kcps} / \mathrm{MBq} /{ }^{\circ} \mathrm{C}=10 \% /{ }^{\circ} \mathrm{C}, \mathrm{R}=-0.96\right)$ temperature changes. Count rate evaluation showed that although the total count rate is consistently higher at $21^{\circ} \mathrm{C}$ than at $24{ }^{\circ} \mathrm{C}$ for different source activity concentrations, this is mainly due to an increase in scattered and random coincidences. The peak total count rate is $400 \mathrm{kcps}$ at both temperatures but is reached at lower activity at $21^{\circ} \mathrm{C}$. The peak true count rate is $138 \mathrm{kcps}$ (at $100 \mathrm{MBq}$ ) at $21^{\circ} \mathrm{C}$ and $180 \mathrm{kcps}$ (at $125 \mathrm{MBq}$ ) at $24^{\circ} \mathrm{C}$. The peak noise equivalent count rate is also lower at $21^{\circ} \mathrm{C}(70 \mathrm{kcps}$ at $70 \mathrm{MBq})$ than at $24{ }^{\circ} \mathrm{C}(100 \mathrm{kcps}$ at $100 \mathrm{MBq})$. At realistic activity levels, the scatter fraction is lower at higher temperatures, but at the cost of a strong decrease in true count rate.

Conclusions: A model was proposed for the temperature dependence of APD-based PET scanners and evaluated using the LabPET small animal PET scanner. System sensitivity and count rate performance are strongly dependent on ambient temperature while system resolution is not. The authors' results indicate that it is important to assure stable ambient temperature to obtain reproducible results in imaging studies with APD-based PET scanners. () 2013 American Association of Physicists in Medicine. [http://dx.doi.org/10.1118/1.4819823]

Key words: PET, APD, temperature, quantification

\section{INTRODUCTION}

A positron emission tomography (PET) detector usually consists of two components: a scintillation crystal, which converts a single gamma photon to a number of scintillation photons, and a photodetector, which converts the scintillation photons to an electronic pulse. ${ }^{1}$ Photomultiplier tubes (PMTs) are most widely used as photodetectors in PET scanners. More recently solid state detectors such as avalanche photodiodes (APDs) have been introduced as photodetectors for PET. ${ }^{2,3}$ APDs have two important advantages over PMTs. They are insensitive to magnetic field variations, which makes it possible to use them in combined PET-MRI scanners. ${ }^{4-6}$ They are also considerably smaller than PMTs, making it easier to use them in small animal PET scanners and enabling one-to-one coupling between crystal and photodetector. ${ }^{2,7}$

A disadvantage of APDs is the strong dependence of their gain factor on ambient temperature. ${ }^{8}$ This is explained by increased lattice vibrations at higher temperatures, which in turn lead to a higher probability of electron-phonon interactions and a lower avalanche yield. ${ }^{9,10}$ The same mechanism also leads to an increase in breakdown voltage with higher temperature, as more electron-phonon interactions lead to a higher threshold to obtain the avalanche effect. A variation in 
gain of $2 \%-4 \%$ per ${ }^{\circ} \mathrm{C}$ can be observed in most APDs. Apart from a decrease in gain, the dark current is also increased with increasing temperature, leading to more background noise. Overall, the signal to noise ratio of APDs is therefore lowered at higher temperatures.

Due to the strong dependence of APD performance on ambient temperature, the performance of APD-based PET scanners may also be significantly influenced by temperature variations. As temperature fluctuations may occur on relatively short term (within minutes), it is possible that scanner properties such as sensitivity and resolution fluctuate during the day or even during a single PET scan. This may lead to suboptimal data acquisition. Knowledge of detector properties is also needed for accurate modeling in iterative image reconstruction. If detector properties change between different PET acquisitions (or during a single acquisition) and these changes are not incorporated in the image reconstruction algorithm, this will lead to reduced image quality.

The performance of scintillation crystals is also known to be dependent on temperature. ${ }^{11}$ For most types of crystals, a decrease in light yield is observed at higher temperatures. The magnitude of this effect has been quantified for some crystal materials. ${ }^{12}$ For LSO, a decrease in scintillation light output of approximately $1 \%$ per ${ }^{\circ} \mathrm{C}$ can be observed.

The necessity of ensuring ambient temperature stability for APD-based PET scanners is well known. Nevertheless, there have been only few investigations into the effect of temperature on the performance of APD-based PET scanners. Spanoudaki et al. have investigated the effect of temperature on the performance of individual APD-based PET detectors. ${ }^{13}$ They concluded that increasing temperature leads to significant changes in the energy spectrum obtained with the detector. They also demonstrated that this effect is due to a combination of the temperature sensitivity of the APD gain, light yield of the scintillation crystal, and of the electronic noise. As the context of that paper was to determine the requirements for the cooling system of a scanner based on APD detectors, they did not investigate the effect of ambient temperature on characteristics of a complete PET system, such as resolution and sensitivity. In this paper, we present the evaluation of the temperature dependence of the LabPET APD-based small animal PET scanner. We have investigated the effect of temperature on the energy spectrum, sensitivity, resolution, scatter fraction, count rate performance, and image quality of this scanner. Simulations were performed as well as experiments. A theoretical basis for the temperature dependence is proposed which can also be applied to other APD-based PET scanners.

\section{MATERIALS AND METHODS}

\section{A. Hardware}

All acquisitions described in this paper were performed on the Triumph II system (Gamma Medica - Ideas, Sherbrooke, Canada). This is a trimodality system consisting of a micro-PET (LabPET8), micro-CT (X-O), and micro-SPECT (X-SPECT). The LabPET and X-O were used for these ex- periments. The LabPET is an APD-based micro-PET scanner with 50\% LYSO and 50\% LGSO crystals. Virtual one-to-one coupling between crystal and photodetector is achieved by using Phoswich detectors in which each APD is coupled to an LYSO and an LGSO crystal in subsequent axial rings and the different timing properties of both scintillators are used to separate light pulses coming from both crystals. Contrary to designs where Phoswich detectors are used to discriminate depth-of-interaction, ${ }^{14}$ they are used here to discriminate subsequent axial crystals coupled to a single APD. Crystals are organized in detector modules of two (axial) by four (tangential) crystals. Sixteen $(4$ axial $\times 4$ tangential) modules are grouped into a detector cassette, which are repeated 12 times in tangential direction to obtain rings with 192 crystals. The LabPET8 has 4 cassettes in the axial direction, leading to 32 axial crystals and an axial FOV of $7.6 \mathrm{~cm}$. The design and signal processing architecture of the scanner have been extensively described. ${ }^{15,16}$ Evaluation of its performance has also been published elsewhere. ${ }^{17,18}$ Of special interest to this work is the cooling system of the scanner, which is done by drawing ambient air from the bottom of scanner and blowing it toward the top, where ventilators help in the removal of hot air.

During all experiments the ambient temperature was monitored. This was done using a USB temperature logger (THL2 Temperature/Humidity USB Datalogger, UEI Test Instruments, Beaverton, USA). Samples were taken every minute. The temperature logger was always placed at the same location in the center of the scanner room, at about $1 \mathrm{~m}$ from the bore of the scanner. During one experiment, the temperature of the detectors was also recorded. It can be obtained per cassette from the control panel of the scanner.

\section{B. Energy spectrum}

\section{B.1. Theory}

In this section, we present a theoretical framework for the influence of temperature variations on APD-based PET detectors. Spanoudaki et al. ${ }^{13}$ have already discussed the basis of this reasoning in their paper, but it is discussed in more detail here. Before an APD-based PET scanner can be used, the energy spectrum of each detector is calibrated, as depicted in Figs. 1(a)-1(e). When a positron source is inside the field of view (FOV) of the scanner, single gamma photons will be detected by the detectors. For each detected gamma photon, the number of photons produced by the scintillation crystal is proportional to the energy of the gamma photon. Because electric charges are integrated by the electronics of the detector, the amplitude of the pulse produced by the APD upon detection of these scintillation photons will therefore also be proportional to the energy of the gamma photon, albeit within the limits of the energy resolution of the detector. However, the proportionality factor may be different for each detector. When a number of gamma photons are detected by one detector, a pulse amplitude histogram can be created for each detector by binning the amplitudes of detected pulses. Each detector is then calibrated by aligning the peak of this histogram with $511 \mathrm{keV}$. This process can usually be 
(a)

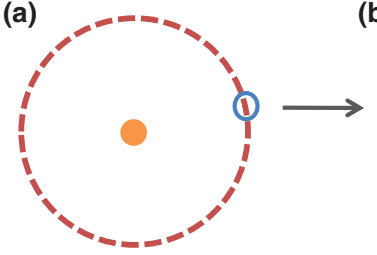

(b)

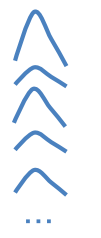

(c)

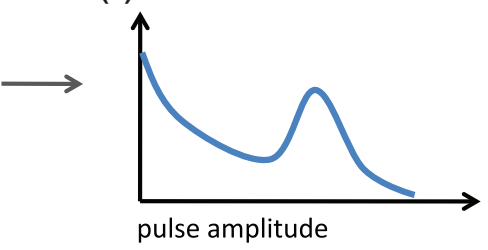

(d)

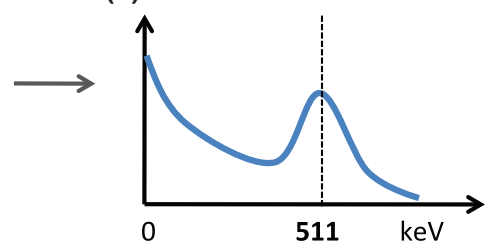

(h)

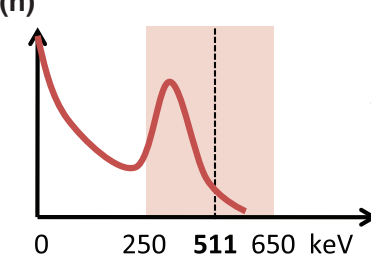

(g)

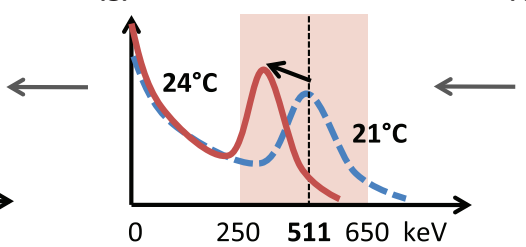

(f)

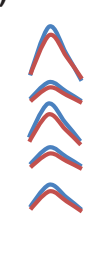

(e)

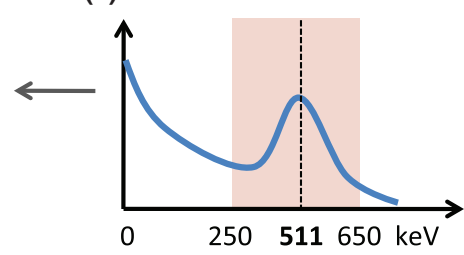

FIG. 1. Illustration of the proposed theoretical model for the temperature dependence of APD-based PET scanners. (a) A positron source is placed inside the FOV of the scanner for calibration. (b) In a single detector of the system, each gamma photon that is detected will give rise to an electronic pulse, whose amplitude is proportional to the energy of the detected gamma photon. (c) After acquiring a large number of gamma photons, a histogram of the pulse amplitudes can be made for each detector. (d) The peak of the pulse amplitude histogram can be aligned with $511 \mathrm{keV}$, whereas a zero pulse amplitude corresponds to 0 keV. As the relationship between pulse amplitude and the energy of the detected gamma photon is linear, we can now determine the corresponding gamma photon energy for each pulse amplitude. (e) At acquisition time, an energy window is applied to all detected gamma photons to remove excess scatter. Here the LabPET energy window of $250-650 \mathrm{keV}$ is shown. (f) When the ambient temperature is increased, the gain of the APD and light yield of the scintillation crystal are reduced. Therefore the pulse amplitude corresponding to a certain gamma photon energy will be smaller. (g) This leads to a scaling of the energy histogram along the energy axis (compression). (h) If the energy window is not recalibrated to the new temperature, a different fraction of photons will be retained within the energy window than at the reference temperature.

performed in an automated manner. After calibration, the same energy window can be applied to all detectors to remove excess scattered coincidences.

Calibration is mostly performed at standard ambient temperature, e.g., $21^{\circ} \mathrm{C}$. For this temperature, it is then known for each detector which gamma photon energy corresponds to a certain pulse amplitude. However, when the ambient temperature changes, the gain factor of the APD is changed as well as the scintillation light yield of the crystal. Hence, the detection of a gamma photon with a certain energy will produce a different pulse amplitude than is expected at the calibration temperature [Fig. 1(f)]. As this change is proportional to the energy of the photon, this will lead to a uniform scaling of the energy spectrum along the energy axis, as illustrated in Fig. 1(g). When temperature is increased APD gain and scintillation light yield decrease and the spectrum is "compressed" along the energy axis. This scaling factor is proportional to the temperature difference and the temperature coefficients of the APD and the scintillator. If the same energy window is applied as at the standard ambient temperature, without recalibration of the relationship between pulse amplitude and photon energy, a different subset of the detected photons will be retained [Fig. 1(h)]. When interpreting the effect of increased temperature, the photopeak is shifted toward the lower limit of the energy window and a wider range of energies are included within the energy window due to the compression along the energy axis. The inverse happens at lower temperatures: the energy spectrum is expanded along the energy axis, yielding an apparent shift of the photopeak toward the upper limit of the energy window and a smaller range of energies included in the energy window.

\section{B.2. Acquisition}

To investigate the effect of temperature changes on the energy histogram of the LabPET scanner, single detector energy histograms were acquired at $21^{\circ} \mathrm{C}$ and $25^{\circ} \mathrm{C}$. At each temperature, two histograms were obtained: a blank acquisition with no source in the FOV and an acquisition with a $1 \mathrm{ml}$ ${ }^{18} \mathrm{~F}$ source with an activity of approximately $37 \mathrm{MBq}$ in the FOV. The blank histograms are needed because the LGSO and LYSO crystals used in the LabPET scanner possess intrinsic radioactivity due to the presence of the Lutetium, of which $2.6 \%$ appears naturally as ${ }^{176} \mathrm{Lu}$, a $\beta^{-}$emitting isotope. By subtracting both energy histograms the counts obtained from the background ${ }^{176} \mathrm{Lu}$ radiation are removed and a single energy histogram is obtained for each temperature, representing only the energy distribution of the point source. For each detector, the energy of the photopeak and the energy resolution were determined at both temperatures. The energy resolution was defined as the full width at half maximum of the photopeak divided by the photopeak energy. The relative change in photopeak energy and energy resolution was then calculated for each detector. For the purpose of visualization, these values were averaged per detector cassette.

\section{C. Sensitivity}

For all simulations and acquisitions described in this section, the same source was used: a calibrated ${ }^{22} \mathrm{Na}$ point source in the center of a $10 \times 10 \times 10 \mathrm{~mm}^{3}$ acrylic cube, conforming to the NEMA NU 4-2008 protocol. ${ }^{19}$ At the start of the experiments, the activity of the source was $255 \mathrm{kBq}$. Unless 
mentioned otherwise, the point source was placed in the center of the FOV.

\section{C.1. Simulation}

Based on the theoretical considerations presented above, a change in temperature will thus lead to a compression or expansion of the energy spectrum at higher or lower temperatures, respectively. The scaling of the energy values depends on the temperature variation and the temperature coefficient of the APD and the scintillation light yield. Although there is no data on the temperature dependence of the scintillation light yield of the LYSO and LGSO crystals used in the LabPET scanner, these data are available for the comparable material LSO. For LSO, the light yield decreases approximately linearly with increasing temperature. This effect therefore works in the same direction as the APD gain temperature dependence, and both effects can be included in a single temperature coefficient of the complete detector, defined as

$$
\tau=\frac{1}{M} \frac{d M}{d T},
$$

where $M$ is the amplitude of the detected pulse and $T$ is the ambient temperature. $\tau$ is expressed as a percentage and is negative. For a temperature difference $\Delta T$ from the reference temperature, the scaling of the energy can be calculated as

$$
E_{\text {new }}=(1+\tau) \Delta T E_{\text {ref }},
$$

where $E_{\text {ref }}$ is the actual energy of the detected photon and $E_{\text {new }}$ is the energy that will be attributed to the detected photon at a temperature differing from the reference temperature by a difference $\Delta T$.

Using this equation, we have performed a simulation of the variation of system sensitivity for different temperatures and different temperature coefficients of the APDs. $21^{\circ} \mathrm{C}$ was used as reference temperature and the simulated temperature range was $18^{\circ} \mathrm{C}$ to $26^{\circ} \mathrm{C}$, in steps of $0.5^{\circ} \mathrm{C}$. Detector temperature coefficients of $-1 \%,-2 \%,-4 \%$, and $-6 \%$ were used. The simulation was performed in three steps. First, a Monte Carlo simulation was performed of an acquisition of the ${ }^{22} \mathrm{Na}$ point source using GATE and a model of the LabPET scanner. ${ }^{20,21}$ The simulated energy resolution was $25 \%$ at $511 \mathrm{keV}^{18}$ No energy window was set on this simulation, and hence the full energy histogram was extracted. Subsequently, for each temperature point and temperature coefficient, the energy spectrum was scaled using Eq. (2). Then the standard LabPET energy window was applied by calculating the integral between the points in the histogram which correspond to $250 \mathrm{keV}$ and $650 \mathrm{keV}$ at the reference temperature. To obtain the relative sensitivity this value was then divided by the integral at the reference temperature.

\section{C.2. Short term variations}

The immediate effect of temperature variations, occurring, e.g., during a single acquisition, was investigated by performing a long experiment with the ${ }^{22} \mathrm{Na}$ point source while ambient temperature was varied considerably. The total time of the experiment was approximately $5 \mathrm{~h}$. Ambient temperature was varied between $21^{\circ} \mathrm{C}$ and $28^{\circ} \mathrm{C}$ by changing air conditioner settings. The experiment started at $21^{\circ} \mathrm{C}$. The air conditioner settings were not changed for $1 \mathrm{~h}$. Then the air conditioner was turned off for $2 \mathrm{~h}$, during which the ambient temperature increased to approximately $28^{\circ} \mathrm{C}$. Finally, the air conditioner was switched on at maximum power again for $2 \mathrm{~h}$, yielding a decrease of ambient temperature until approximately $21^{\circ} \mathrm{C}$. During the experiment, 30 acquisitions of 10 min were performed, and for each frame the sensitivity was calculated based on the NEMA NU 4-2008 protocol, ${ }^{19}$ including the ${ }^{22} \mathrm{Na}$ branching ratio. The correlation coefficient between temperature and sensitivity was calculated. Linear regression was used to determine the relationship between both. During this experiment, the temperature per detector cassette, as available from the scanner control panel, was also recorded at the end of each acquisition. For each cassette, linear regression was performed to determine the relationship between ambient temperature and detector temperature.

\section{C.3. Long term variations}

There may be a difference between the immediate effect of quickly varying temperatures as investigated above, and the effect of temperature changes that have occurred at a certain time point and stabilized for a period of time (hours). We will refer to these temperature changes as long term variations. They may be observed when the baseline temperature of the scanner room is changed, for example because of a change in air conditioner settings, and remains stable at the new temperature. This was investigated by performing acquisitions of the ${ }^{22} \mathrm{Na}$ point source at different temperatures after they have stabilized. In total 13 acquisitions were performed at temperatures between $21^{\circ} \mathrm{C}$ and $25^{\circ} \mathrm{C}$. Before each acquisition the temperature in the scanner room had stabilized for at least $4 \mathrm{~h}$. This was verified by checking the temperature log and assuring that temperature variations in the preceding $4 \mathrm{~h}$ were smaller than $\pm 0.1{ }^{\circ} \mathrm{C}$. The acquisition time was $5 \mathrm{~min}$. The data were processed as in the previous experiment to obtain the peak sensitivity of the system in each acquisition and determine the relationship between sensitivity and temperature.

\section{C.4. NEMA axial sensitivity profile}

In a last sensitivity experiment, the axial sensitivity profile of the scanner was measured at $21^{\circ} \mathrm{C}$ and $24^{\circ} \mathrm{C}$. At each temperature, an experiment was performed according to the NEMA NU 4-2008 protocol. ${ }^{19}$ Sensitivity was measured in the transaxial center of the field of view along the axial direction in steps of $2 \mathrm{~mm}$. An acquisition of $1 \mathrm{~min}$ was performed at each location. The data at each point were processed as described above. Blank scans were also acquired at the same temperatures and used to subtract background counts from the acquisitions. To assess the reproducibility of this experiment, the experiment was repeated three times at $21^{\circ} \mathrm{C}$. As the reproducibility at $21^{\circ} \mathrm{C}$ was very high, only one experiment was performed at $24^{\circ} \mathrm{C}$. 


\section{D. Spatial resolution}

System spatial resolution was evaluated at $21^{\circ} \mathrm{C}$ and $24^{\circ} \mathrm{C}$ using the NEMA NU 4-2008 protocol. The ${ }^{22} \mathrm{Na}$ point source described above was placed in the center of the axial field of view and at radial distances of $5,10,15$, and $25 \mathrm{~mm}$ from the transaxial center of the field of view. The same measurements were done at $1 / 4$ of the axial field of view. The acquisition time for all scans was 3 min. Images were reconstructed using 2D filtered back projection with single slice rebinning including all oblique slices, using the high-resolution protocol $(0.25 \mathrm{~mm}$ isotropic voxel size) implemented on the scanner. For each acquisition, the full width at half maximum (FWHM) and full width at tenth maximum (FWTM) of the axial, radial, and tangential resolutions was determined as defined in the protocol.

\section{E. Scatter fraction, count losses, and random coincidences}

\section{E.1. Energy histogram composition}

As temperature changes have an effect on the acquired counts by scaling the energy histogram, and unscattered and scattered photons have a different energy spectrum, the effect of temperature changes on the detection of scattered and unscattered counts will be different. To illustrate this, we have estimated the energy distribution of scattered and unscattered photons by performing a GATE simulation of the NEMA ratsize phantom with the LabPET scanner. The energy resolution of the scanner was set to $25 \%$ at $511 \mathrm{keV}^{18}$ The ratsize phantom consists of a HDPE (high density polyethylene - density $0.96+/-0.1 \mathrm{~g} / \mathrm{cm}^{3}$ ) cylinder with diameter $50 \mathrm{~mm}$ and length $150 \mathrm{~mm}$. At a radial distance of $17.5 \mathrm{~mm}$ from the center of the cylinder a cylindrical hole with $3.2 \mathrm{~mm}$ diameter is drilled in which the activity is placed. A total activity of $10 \mathrm{MBq}$ of ${ }^{18} \mathrm{~F}$ was simulated for $4 \mathrm{~s}$ to obtain sufficient counts. From the coincidences separate energy histograms were obtained for true, scattered, and random coincidences.

\section{E.2. Count rate variation at stable temperature}

To evaluate the influence of temperature on the scatter fraction and count rate performance over a wide range of count rates, the corresponding experiment defined in the NEMA NU 4-2008 standard was performed at $21^{\circ} \mathrm{C}$ and $24^{\circ} \mathrm{C}$. The stability of the temperature was verified as described above. The NEMA rat-size phantom, described in Sec. 2.E.1, was used. ${ }^{11} \mathrm{C}$ was used as radioactive source. At the start of the experiment the total activity inside the phantom was $325 \mathrm{MBq}$ and $350 \mathrm{MBq}$ at $21^{\circ} \mathrm{C}$ and $24^{\circ} \mathrm{C}$, respectively. A scan was performed with a total duration of $180 \mathrm{~min}$, acquiring data during decay of the source to approximately $1 \mathrm{MBq}$ at both temperatures. The data were split into 18 frames of $10 \mathrm{~min}$ and prompt and delayed histograms were extracted for each frame. In each frame, the total, true, scattered, random, and noise equivalent count rates were calculated using the methodology defined in the NEMA NU 4-2008 protocol.

\section{E.3. Temperature variation at constant low count rate}

The experiment described in Sec. 2.E.2 will yield information on performance of the system over a wide range of count rates, but only at $21{ }^{\circ} \mathrm{C}$ and at $24^{\circ} \mathrm{C}$. It would be interesting to investigate the performance over a range of temperatures. Another remark on the previous experiment is that the scanner electronics may generate considerable heat at the start of the experiment by acquiring at such high count rates. This may lead to a temperature effect which is due to the nature of this acquisition and would not occur in a realistic imaging situation. For these reasons, two experiments were also performed with the NEMA rat-size phantom at low count rates and at different temperatures. This was done by inserting a $7.6 \mathrm{~cm}$ long ${ }^{68} \mathrm{Ge}$ rod source with an activity of $11.7 \mathrm{MBq}$ into the rat-size phantom. In the first experiment, the ambient temperature was first kept constant at $21^{\circ} \mathrm{C}$ for $1 \mathrm{~h}$. Then the air conditioner was switched off for $2 \mathrm{~h}$, leading to an ambient temperature of approximately $28^{\circ} \mathrm{C}$. The air conditioner was then switched back on at maximum power for $2 \mathrm{~h}$, yielding a return to a temperature of approximately $21^{\circ} \mathrm{C}$. This evolution of temperature is comparable to the one obtained in the short term sensitivity experiment. The data were split into 30 frames of $10 \mathrm{~min}$ and processed as described above. During a second experiment, the air conditioner settings were changed in steps from $21^{\circ} \mathrm{C}$ to $25^{\circ} \mathrm{C}$ and back to $22^{\circ} \mathrm{C}$ and the temperature was allowed to stabilize for $30 \mathrm{~min}$ after each change in air conditioner settings. In that experiment, 8 acquisitions of $10 \mathrm{~min}$ were performed and processed as described above.

\section{F. Image quality}

To investigate the effect of temperature in a realistic imaging situation, several acquisitions were performed of the NEMA image quality phantom, as described in the NEMA NU 4-2008 standard. The cylindrical image quality phantom consists of three sections which are used to evaluate nonuniformity, recovery coefficients for warm rods of different diameters and spill over ratio of warm background activity into cold regions. A total of six acquisitions of the phantom were performed, with stable temperatures of $21^{\circ} \mathrm{C}$ and $24^{\circ} \mathrm{C}$ on alternating days. The phantom was filled with approximately $7 \mathrm{MBq}$ of ${ }^{18} \mathrm{~F}$-FDG and the acquisition time was $20 \mathrm{~min}$ as defined in the protocol. A CT was also obtained for attenuation correction. CT settings were $285 \mu \mathrm{As}$ and $75 \mathrm{kVp}$ and $\mathrm{CT}$ images were reconstructed to a $512 \times 512 \times 512$ matrix with $0.1 \mathrm{~mm}$ isotropic voxel size using the software provided by the manufacturer. After each acquisition a normalization scan with a rotating ${ }^{68} \mathrm{Ge}$ rod source was performed.

Two reconstructions were performed for each acquisition: one with the correct normalization scan (acquired directly after the phantom acquisition) and one with the average normalization scan acquired at the other temperature. The average normalization scan at a temperature was obtained by calculating the average number of counts in the three normalization scans acquired at that temperature in each bin. PET images were reconstructed using the maximum 


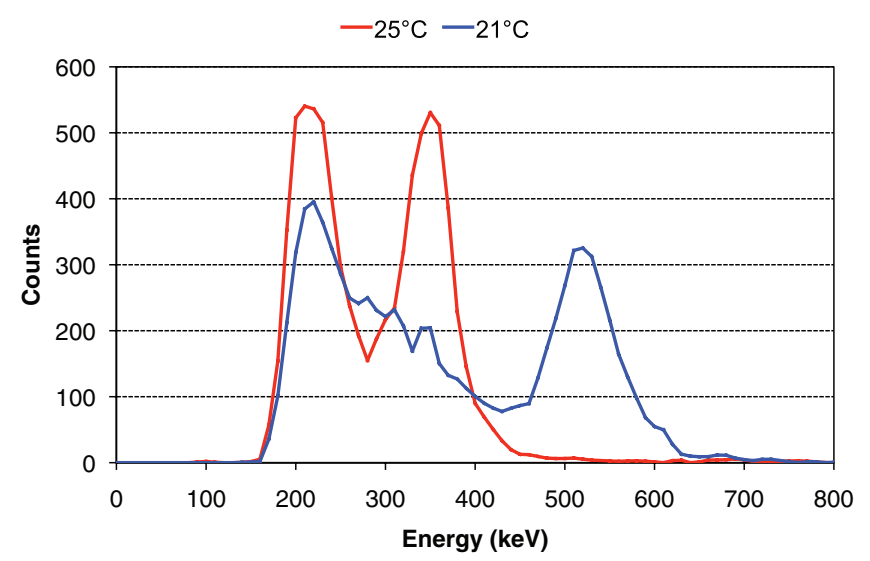

FIG. 2. Energy histograms acquired on a single LGSO detector of the LabPET system at $21^{\circ} \mathrm{C}$ and $25^{\circ} \mathrm{C}$.

likelihood - expectation maximization (ML-EM) algorithm with CT-based attenuation correction. The reconstructed matrix was $160 \times 160 \times 128$ with $0.5 \mathrm{~mm}$ isotropic voxel size. Postsmoothing with a Gaussian filter with FWHM of $1 \mathrm{~mm}$ was performed. Nonuniformity, recovery coefficients, and spill-over-ratio's were calculated in each reconstructed image as described in the protocol.

\section{RESULTS}

\section{A. Energy histogram}

The energy histograms of a single LGSO detector acquired at $21^{\circ} \mathrm{C}$ and $25^{\circ} \mathrm{C}$ are shown in Fig. 2. The peak of the en-
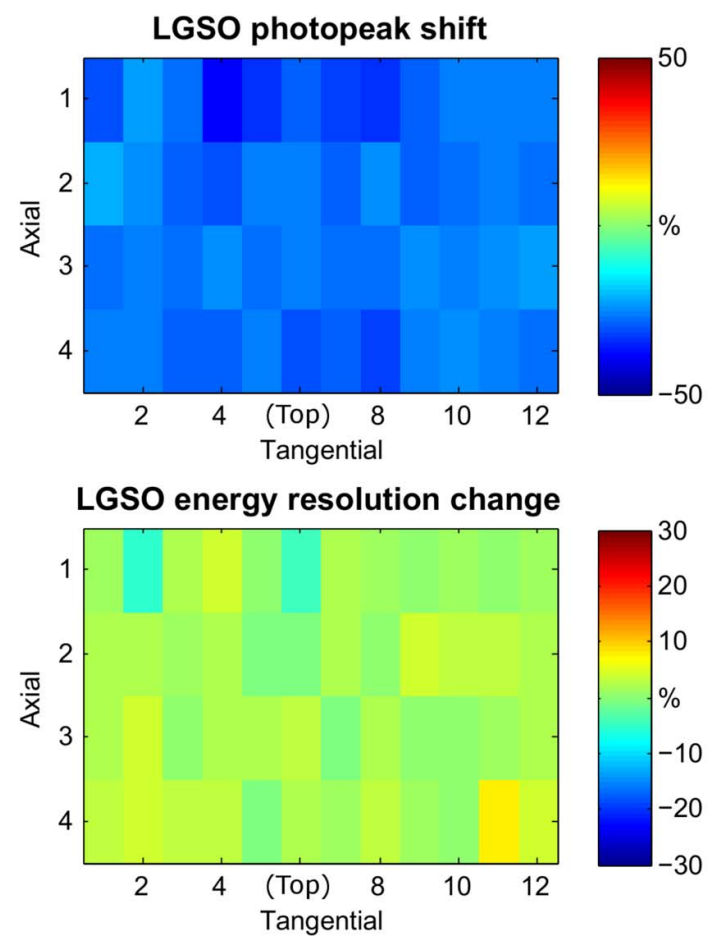

ergy histogram acquired at $21^{\circ} \mathrm{C}$ is located approximately at $511 \mathrm{keV}$. At $25^{\circ} \mathrm{C}$, the peak is located approximately at $350 \mathrm{keV}$. This is a relative shift of the energy histogram to the left over $-31 \%$ or $-7.8 \%$ per ${ }^{\circ} \mathrm{C}$ and essentially changes the $250-650 \mathrm{keV}$ energy window to a 328-852 keV energy window. Each energy bin also contains more counts at $25^{\circ} \mathrm{C}$ than at $21^{\circ} \mathrm{C}$ as each bin at $25^{\circ} \mathrm{C}$ contains a wider energy range due to compression of the energy spectrum. Figure 3 shows the average relative shift of the photopeak position and average change in energy resolution between both temperatures per cassette. The average photopeak shift from $21^{\circ} \mathrm{C}$ to $25^{\circ} \mathrm{C}$ for LGSO and LYSO was $-27.4 \pm 7.1 \%(6.85 \pm 1.78 \%$ per $\left.{ }^{\circ} \mathrm{C}\right)$ and $-20.1 \pm 10.1 \%\left(5.1 \pm 2.53 \%\right.$ per $\left.{ }^{\circ} \mathrm{C}\right)$, respectively. The average change in energy resolution was $+1.8 \pm 5.2 \%$ and $+1.9 \pm 9.7 \%$, respectively.

\section{B. Sensitivity}

\section{B.1. Simulation}

The results of the simulation of system sensitivity for different temperatures and different detector temperature coefficients are shown in Fig. 4. When the temperature is increased relative to the reference temperature, an approximately linear decrease in sensitivity is observed. Over the temperature interval between $21^{\circ} \mathrm{C}$ and $24{ }^{\circ} \mathrm{C}$, a relative sensitivity decrease of $-5 \%,-10 \%,-21 \%$, and $-26 \%$ is seen for temperature coefficients of $1 \%, 2 \%, 4 \%$, and $6 \%$, respectively. This corresponds to respective decreases of $-1.7 \%,-3.3 \%$, $-7 \%$, and $-8.7 \%$ per ${ }^{\circ} \mathrm{C}$. When temperature is increased further, the relationship becomes nonlinear for high temperature
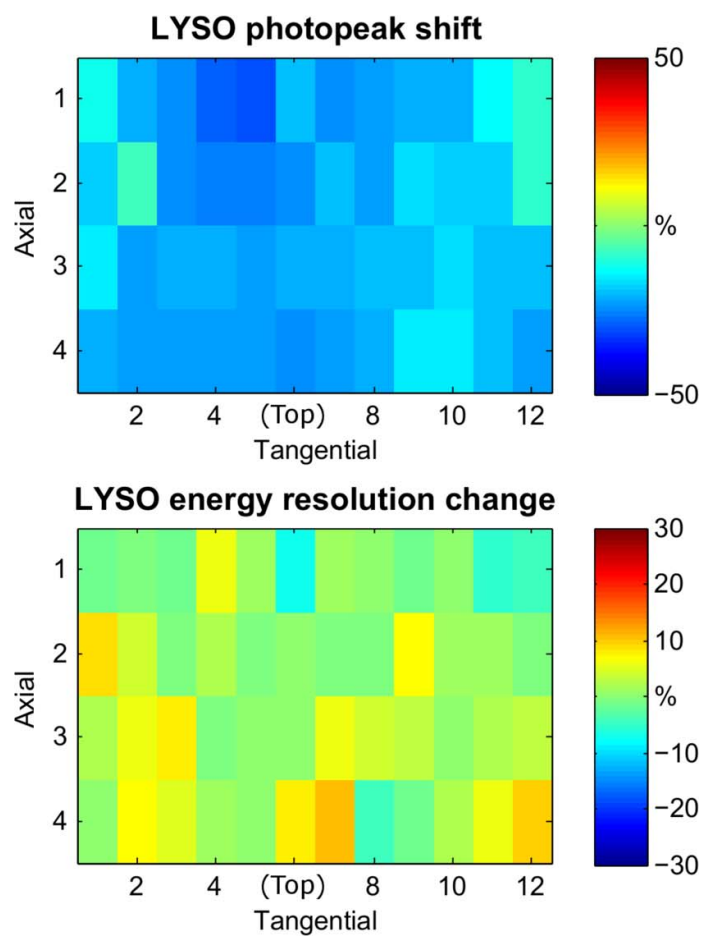

FIG. 3. Relative shift of the photopeak and relative change of the energy resolution in LGSO and LYSO crystals when changing the ambient temperature from $21^{\circ} \mathrm{C}$ to $25^{\circ} \mathrm{C}$. One pixel represents a detector cassette (four modules axial by four modules tangential). In the tangential direction the modules at the top of the scanner ring are depicted in the middle. 


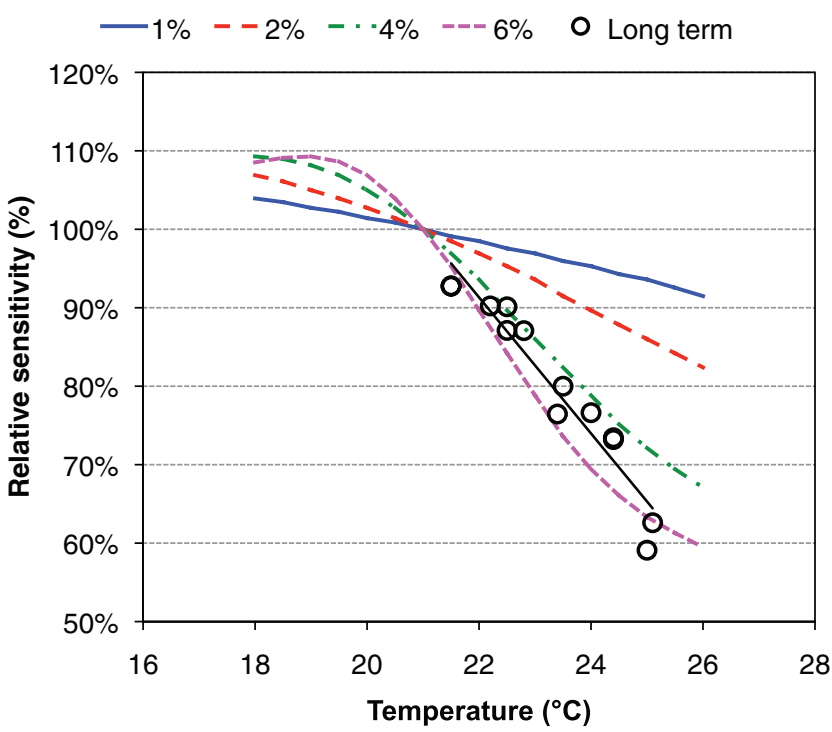

FIG. 4. Simulation of the sensitivity of an APD-based PET scanner at different temperatures and for different APD temperature coefficients. The sensitivity was calculated relative to the sensitivity at a reference temperature of $21^{\circ} \mathrm{C}$. The results of the measurement of the effect of long term sensitivity changes is also shown, as well as the linear regression on that data set.

coefficients. This is also the case when temperature is decreased from the reference temperature.

\section{B.2. Short term variations}

Figure 5 depicts the variation of ambient temperature, average detector temperature, and sensitivity over time during the scan performed to evaluate the immediate effect of

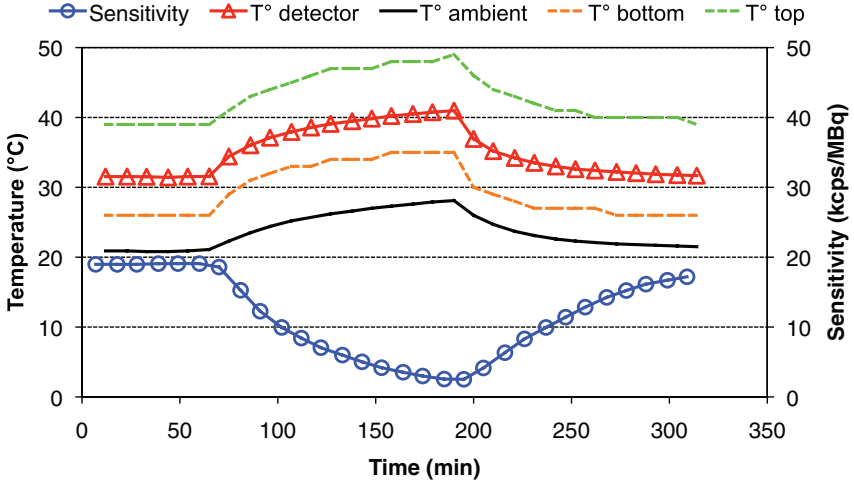

FIG. 5. Peak sensitivity (inside the energy window) of the LabPET system, average detector temperature throughout the system, and ambient temperature during a $5 \mathrm{~h}$ acquisition with varying air conditioner settings. Detector temperature for a cassette at the top and the bottom of the scanner are also shown (dashed lines).

temperature changes. The correlation coefficient of ambient temperature and sensitivity was -0.95 , with a peak sensitivity change of $-2.32 \mathrm{kcps} / \mathrm{MBq}$ per ${ }^{\circ} \mathrm{C}$ or approximately $-12 \%$ per ${ }^{\circ} \mathrm{C}$. The correlation of average detector temperature with ambient temperature was 0.98 . Figure 5 also shows the evolution of detector temperature in a module at the top and at the bottom of the scanner, demonstrating a difference of almost $15^{\circ} \mathrm{C}$ between both modules and a slightly different time course: the module at the top seems to lag behind the module at the bottom. The linear regression analysis between ambient temperature and detector temperature was therefore performed separately in the warming up phase (until approximately $180 \mathrm{~min}$ ) and the cooling down phase (after $180 \mathrm{~min}$ ) of the experiment. Figure 6 shows the correlation coefficient
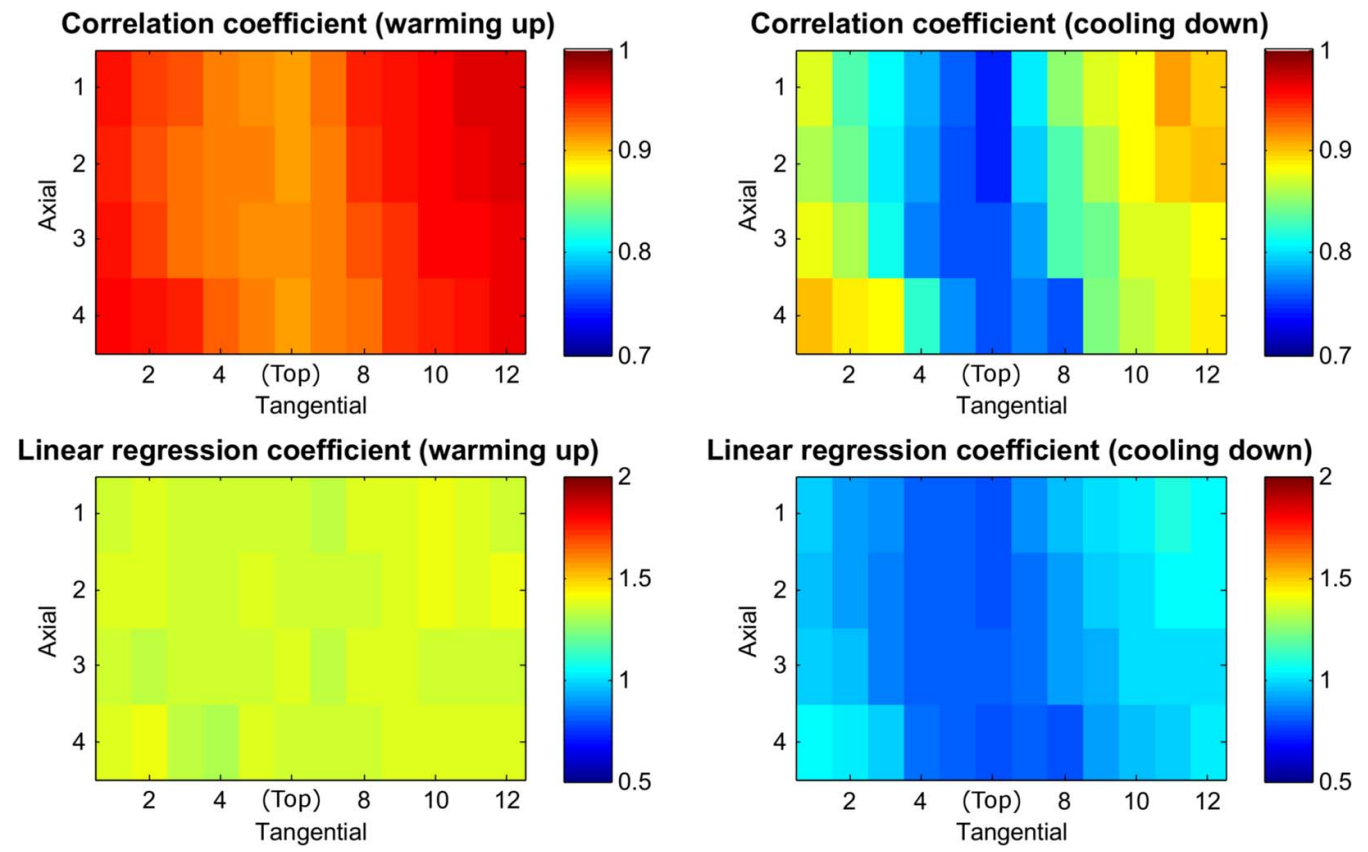

FIG. 6. Spatial distribution throughout the scanner of the correlation coefficient between detector temperatures and ambient temperature as well as the linear regression coefficient of detector temperatures versus ambient temperature during the experiment depicted in Fig. 5. The calculations were split up between the warming up phase (until $180 \mathrm{~min}$ ) and cooling down phase (after $180 \mathrm{~min}$ ). One pixel represents a detector cassette (four modules axial by four modules tangential). In the tangential direction, the cassettes at the top of the scanner ring are depicted in the middle. 


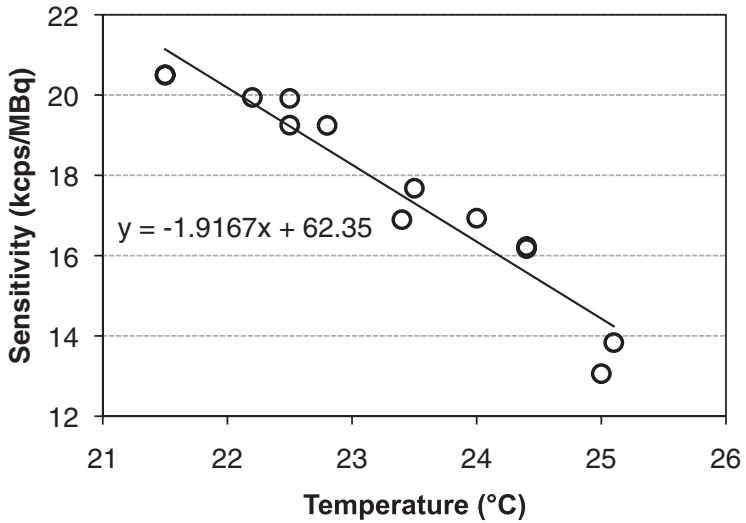

FIG. 7. Peak sensitivity of the LabPET system at different temperatures after stabilization of temperature for at least $4 \mathrm{~h}$. The linear regression line is also shown.

between detector temperature and ambient temperature per cassette for both phases. The linear regression coefficients are also depicted, illustrating the temperature change at the detectors per ${ }^{\circ} \mathrm{C}$ ambient temperature change. In the warming up phase and the cooling down phase, the correlation coefficients are on average $0.94 \pm 0.02$ and $0.83 \pm 0.05$, respectively, but the correlation with detector temperature at the top of the scanner is lower than at the bottom. In the warming up phase, the linear regression coefficients are uniform throughout the scanner, with a temperature rise of $1.37 \pm 0.04{ }^{\circ} \mathrm{C}$ at the detectors per ${ }^{\circ} \mathrm{C}$ ambient temperature increase. During the cooling down phase, a regional effect is seen: the detectors at the bottom of the scanner cool down almost as quickly as ambient temperature, whereas the detectors at the top only cool down $0.7^{\circ} \mathrm{C}$ per ${ }^{\circ} \mathrm{C}$ ambient temperature decrease. The overall average linear regression coefficient during the cooling down phase was $0.92 \pm 0.1{ }^{\circ} \mathrm{C} /{ }^{\circ} \mathrm{C}$.

\section{B.3. Long term variations}

Temperature and sensitivity measured at different time points at stabilized conditions are shown in Fig. 7. The correlation coefficient of temperature and sensitivity was -0.96 . Linear regression revealed an estimated decrease of sensitivity of $-1.92 \mathrm{kcps} / \mathrm{MBq}$ per ${ }^{\circ} \mathrm{C}$ or approximately $8.5 \%$ per ${ }^{\circ} \mathrm{C}$. The measured data points are also plotted in Fig. 4. In that plot the sensitivity in each point is depicted relative to the sensitivity at $21^{\circ} \mathrm{C}$ estimated by linear regression. The regression line determined by linear regression is also plotted.

\section{B.4. NEMA axial sensitivity profile}

The axial sensitivity profile at $21^{\circ} \mathrm{C}$ and $24^{\circ} \mathrm{C}$ is depicted in Fig. 8. A total of three measurements were performed at $21^{\circ} \mathrm{C}$ to verify reproducibility. In the graph the average sensitivity at each point is shown and the error bars depict the standard deviation. The average standard deviation is $0.28 \mathrm{kcps} / \mathrm{MBq}$, reflecting a good reproducibility of the experiment. The peak sensitivity at $21^{\circ} \mathrm{C}$ and $24^{\circ} \mathrm{C}$ was $21.0 \mathrm{kcps} / \mathrm{MBq}$ and $17.0 \mathrm{kcps} / \mathrm{MBq}$ respectively, or a change of sensitivity by $-1.33 \mathrm{kcps} / \mathrm{MBq}$ per ${ }^{\circ} \mathrm{C}$.

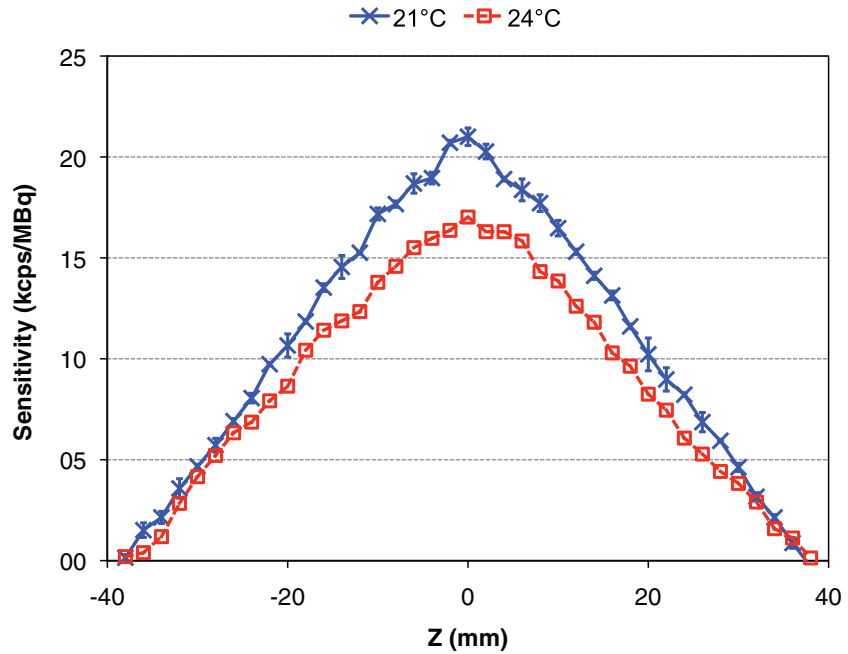

FIG. 8. Axial sensitivity profile of the LabPET system at $21^{\circ} \mathrm{C}$ and $24^{\circ} \mathrm{C}$ measured using the NEMA NU 4-2008 protocol. The average of three measurements is shown for $21^{\circ} \mathrm{C}$, with the error bars depicting one standard deviation. Because of the small variations between the experiments at $21^{\circ} \mathrm{C}$, only one measurement was performed at $24^{\circ} \mathrm{C}$.

\section{C. Spatial resolution}

The FWHM and FWTM of the tangential, radial, and axial resolution in the center of the scanner at different radial distances at $21^{\circ} \mathrm{C}$ and $24^{\circ} \mathrm{C}$ is depicted in Fig. 9. The FWHM of the radial resolution increases from $2.1 \mathrm{~mm}$ to $3.2 \mathrm{~mm}$ at increasing radial distances due to the parallax effect. The FWHM of the tangential resolution is approximately $2.1 \mathrm{~mm}$ at all radial distances and is approximately equal for both temperatures. No difference is observed between both temperatures. The FWHM of the axial resolution is different for both temperatures: at $21^{\circ} \mathrm{C}$ it ranges from $2.8 \mathrm{~mm}$ to $8.1 \mathrm{~mm}$ with increasing radial distance, while at $24^{\circ} \mathrm{C}$ it ranges from $2.8 \mathrm{~mm}$ to $9.7 \mathrm{~mm}$. The relative increase in FWHM of the axial resolution is higher at larger radial distance: $20 \%$ at $25 \mathrm{~mm}$ and $9 \%$ at $10 \mathrm{~mm}$. The FWTMs of the radial and tangential resolution are almost $1 \mathrm{~mm}$ smaller at $24^{\circ} \mathrm{C}$ than at $21^{\circ} \mathrm{C}$. For the axial resolution, this is also the case at $5 \mathrm{~mm}$ radial distance. At larger radial distances, the FWTM of the axial resolution is larger at $24^{\circ} \mathrm{C}$, but the difference with $21^{\circ} \mathrm{C}$ is smaller than for the FWHM. Comparable results were observed at $1 / 4$ th of the axial FOV.

\section{D. Scatter fraction, count losses, and random coincidences}

\section{D.1. Energy histogram composition}

The energy histograms of the true, scattered, and random coincidences obtained by simulating the NEMA rat-size phantom are shown in Fig. 10. The limited energy resolution of the scanner leads to a broad peak around $511 \mathrm{keV}$ for the true coincidences. Nevertheless, the majority of true coincidences are contained within the $250-650 \mathrm{keV}$ energy window set on the scanner. As expected the energy histogram 

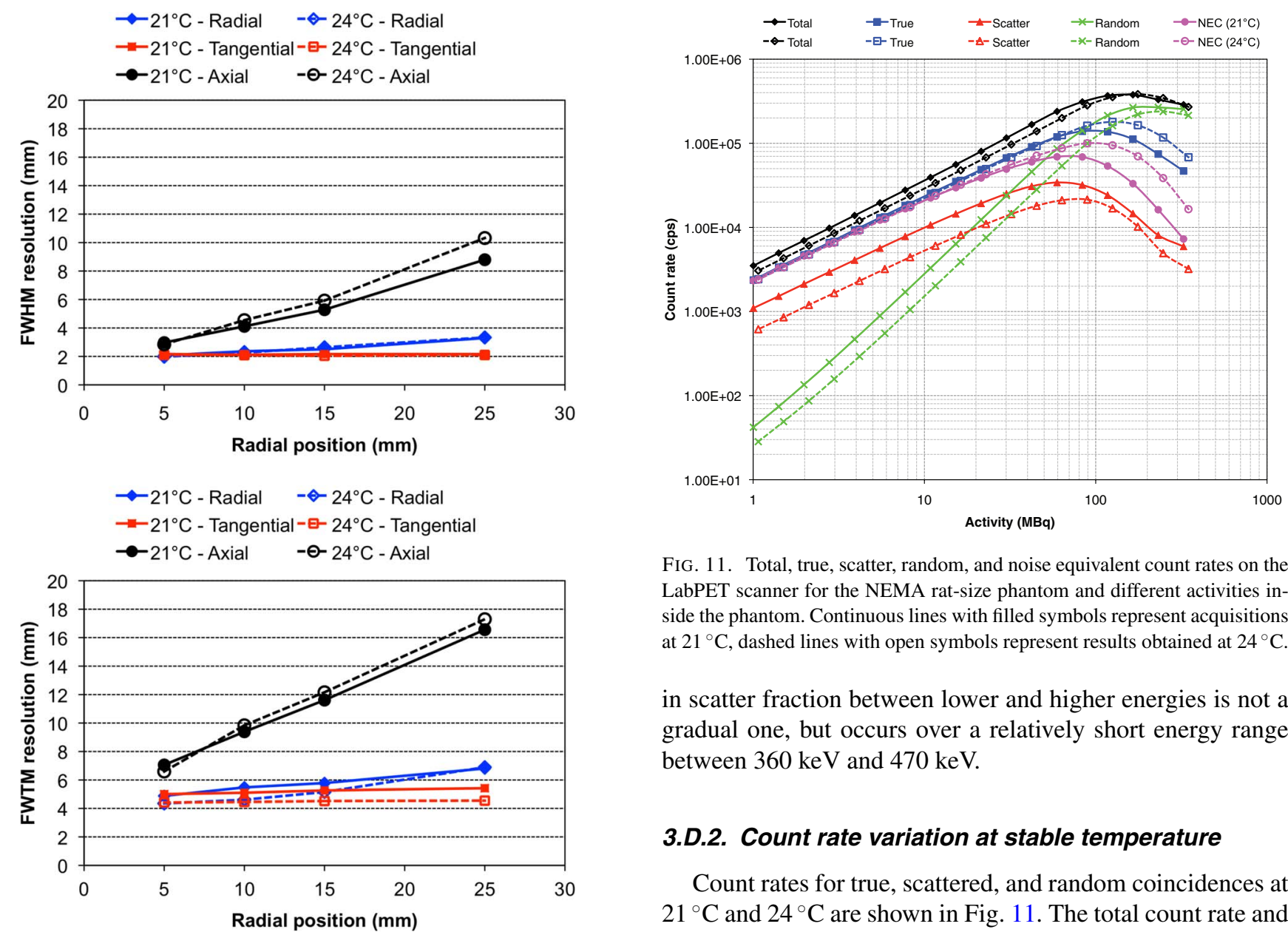

FIG. 11. Total, true, scatter, random, and noise equivalent count rates on the LabPET scanner for the NEMA rat-size phantom and different activities inside the phantom. Continuous lines with filled symbols represent acquisitions at $21^{\circ} \mathrm{C}$, dashed lines with open symbols represent results obtained at $24^{\circ} \mathrm{C}$.

in scatter fraction between lower and higher energies is not a gradual one, but occurs over a relatively short energy range between $360 \mathrm{keV}$ and $470 \mathrm{keV}$.

\section{D.2. Count rate variation at stable temperature}

Count rates for true, scattered, and random coincidences at $21^{\circ} \mathrm{C}$ and $24^{\circ} \mathrm{C}$ are shown in Fig. 11. The total count rate and noise equivalent count rate (NEC) are also shown. The peak true count rate at $21^{\circ} \mathrm{C}$ is reached at $100 \mathrm{MBq}$ and is approximately $138 \mathrm{kcps}$. At $24^{\circ} \mathrm{C}$, the peak true count rate is reached at $125 \mathrm{MBq}$ and is higher $(180 \mathrm{kcps})$. The peak total count rate is $400 \mathrm{kcps}$ at both temperatures but is reached at higher activity at $24^{\circ} \mathrm{C}$. At each activity point below the peak total activity, the total count rate at $21^{\circ} \mathrm{C}$ is higher than at $24^{\circ} \mathrm{C}$. Scattered and random coincidence count rates are consistently higher at $21^{\circ} \mathrm{C}$ at all activity, but the true coincidence rate is only slightly higher at $21{ }^{\circ} \mathrm{C}$ in the low activity range (below $50 \mathrm{MBq}$ ). The combination of these effects also leads to a higher peak NEC rate at $24^{\circ} \mathrm{C}$ : $100 \mathrm{kcps}$ at $100 \mathrm{MBq}$ versus $70 \mathrm{kcps}$ at $70 \mathrm{MBq}$ at $21^{\circ} \mathrm{C}$.

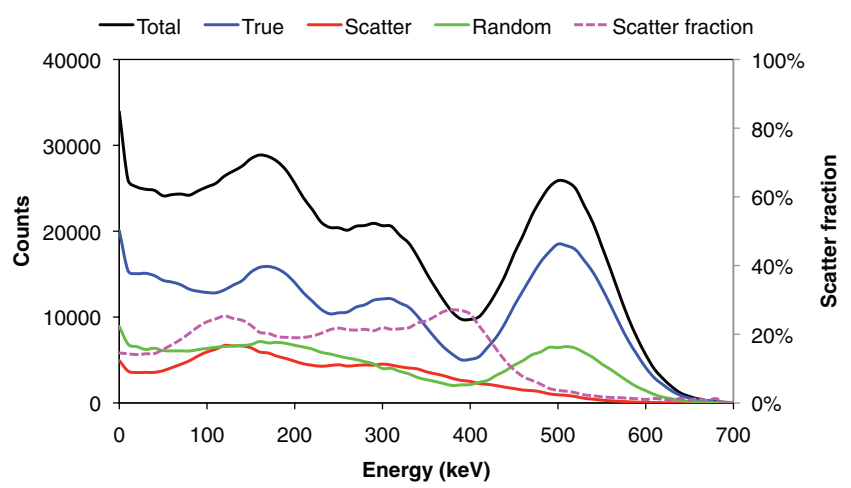

FIG. 10. Simulation of the energy histogram of the LabPET scanner. The total energy histogram is shown as well as the energy histogram of true, scattered, and random coincidences separately. The fraction of scattered photons in each bin is also shown (axis on the right).

\section{D.3. Temperature variation at constant low count rate}

The results from both experiments with the NEMA rat-size phantom at constant low count rates are shown in Fig. 12. The solid lines with filled symbols represent the experiment during which ambient temperature was continuously varying, from $22^{\circ} \mathrm{C}$ to $29^{\circ} \mathrm{C}$ and back again over $5 \mathrm{~h}$. All count rates are reduced at higher temperatures, but there is a different effect on different count rates. The true count rate is reduced by a factor of 14 from $22{ }^{\circ} \mathrm{C}$ to $29^{\circ} \mathrm{C}$, but the scattered count rate is reduced by a factor 21 . There is also a strong impression of hysteresis during this experiment, where higher count rates than expected are seen when warming up and lower count 


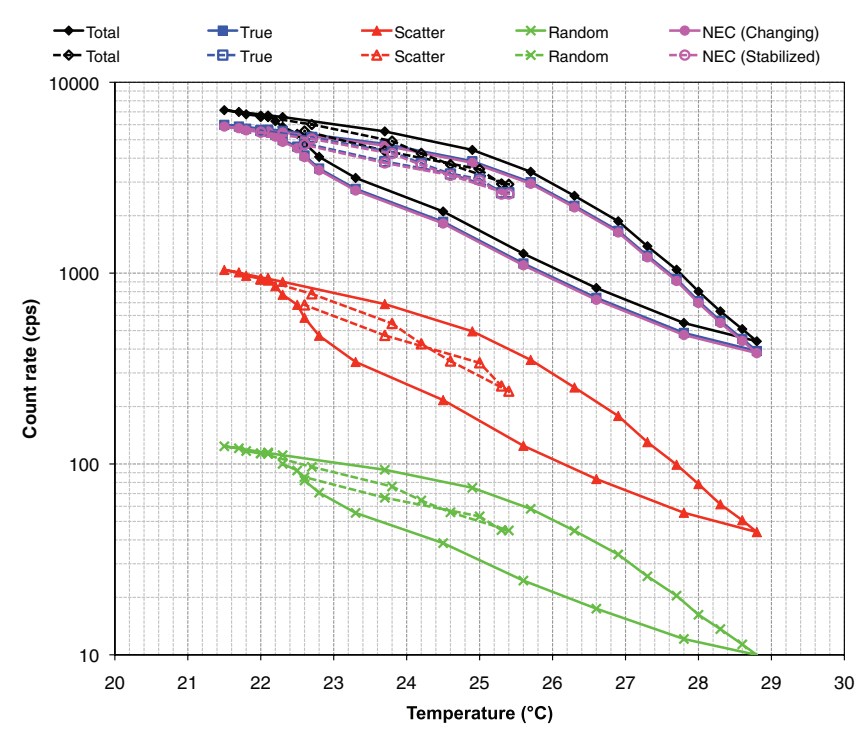

FIG. 12. Total, true, scatter, random, and noise equivalent count rates on the LabPET scanner for the NEMA rat-size phantom with a $11.7 \mathrm{MBq}{ }^{68} \mathrm{Ge}$ source at different temperatures. Continuous lines with filled symbols represent a $5 \mathrm{~h}$ acquisition during which ambient temperature was varied as in the experiment depicted in Fig. 5, with time increasing in clockwise direction on the curves. Dashed lines with open symbols represent acquisitions after stabilization of ambient temperature for $30 \mathrm{~min}$

rates than expected when cooling down. To investigate if this was just a time effect or actual hysteresis of the detectors, the dashed lines represent the results of an experiment during which temperature was stable for 30 min before each acquisition. Those results demonstrate comparable count rates for a specific temperature when warming up as when cooling down.
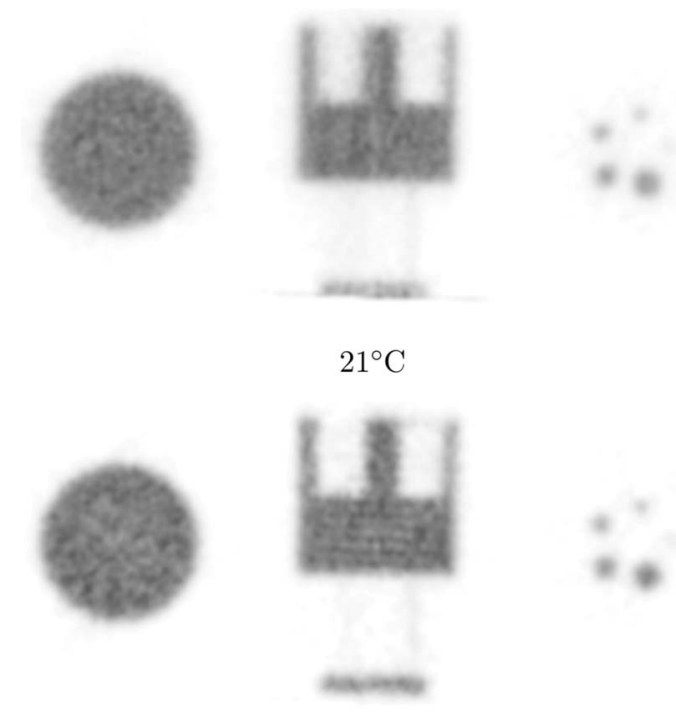

$24^{\circ} \mathrm{C}$

FIG. 13. Different slices from reconstructed PET images of the NEMA image quality phantom at $21^{\circ} \mathrm{C}$ (top) and at $24^{\circ} \mathrm{C}$ (bottom), reconstructed using normalization at the correct temperature. From left to right: a transverse slice through the uniform section, a coronal slice through the uniform section, and two cold cylinders and a transverse slice through the section with warm rods.

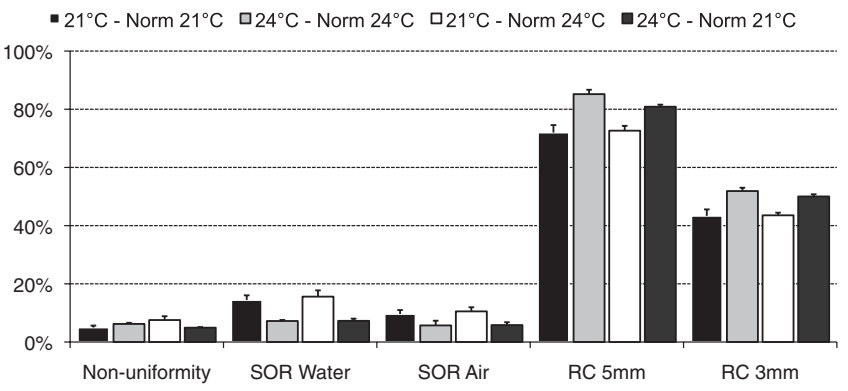

FIG. 14. Results from different scans of the NEMA image quality phantom at $21^{\circ} \mathrm{C}$ and at $24^{\circ} \mathrm{C}$, reconstructed using normalization at either the correct temperature or the incorrect temperature.

\section{E. Image quality}

Two transverse slices and one coronal slice of the reconstructed images of the NEMA image quality phantom acquired at $21{ }^{\circ} \mathrm{C}$ and $24^{\circ} \mathrm{C}$ and reconstructed with the correct normalization scan are shown in Fig. 13. An axial nonuniformity artifact is visible at $24^{\circ} \mathrm{C}$ in the coronal slice, as well as a slightly higher reconstructed activity in the cold cylinders at the top of the phantom at $21^{\circ} \mathrm{C}$. Figure 14 depicts the quantitative results. When using a normalization scan acquired at the same temperature, the spill-over-ratios (SORs) in air and water are approximately twice as large at $21^{\circ} \mathrm{C}$ than at $24^{\circ} \mathrm{C}$. The recovery coefficients (RCs) in the rods with $5 \mathrm{~mm}$ and 3 $\mathrm{mm}$ diameter are also larger at $24^{\circ} \mathrm{C}$. Nonuniformity is higher at $24^{\circ} \mathrm{C}$. Reconstructing using normalization at the incorrect temperature yields comparable results, except for the nonuniformity, which is then larger for the images acquired at $21^{\circ} \mathrm{C}$.

\section{DISCUSSION}

Based on the sensitivity of APD gain and scintillation light yield to temperature and the general signal processing architecture of PET scanners, we have proposed a theoretical model for the temperature dependence of APD-based PET scanners. Comparable results at detector level have previously been described by Spanoudaki et al. ${ }^{13}$ Our hypothesis is that, due to decreased gain of the APDs and decreased scintillation light yield at higher temperatures, the acquired energy histogram of APD-based PET detectors will be compressed along the energy axis relative to the reference energy histogram. If the energy window settings on the scanner are not changed to account for this, a different fraction of detected photons will be discarded compared to the reference temperature. Based on this theoretical model, we performed numerical simulations of the sensitivity of APD-based scanners with different detector temperature coefficients. This showed that first of all an approximately linear relationship between temperature and sensitivity is observed when temperature is increased up to $3{ }^{\circ} \mathrm{C}$ from the reference temperature. When larger temperature differences are simulated the relationship becomes nonlinear. As can be expected, this happens first for the highest temperature coefficients. Second, a decrease of ambient temperature leads to nonlinearities more quickly. At a temperature coefficient of $6 \%$, sensitivity 
increases first when the ambient temperature is decreased, but then decreases again. Finally, although the relationship between temperature and system sensitivity is linear, the relative change in sensitivity per ${ }^{\circ} \mathrm{C}$ is higher than the change in detector gain. All of these observations can be explained by the shape of the energy histogram and the application of the energy window.

During all the experiments performed for this work, ambient temperature was recorded at the same position in the scanner room, and changes in scanner properties were expressed relative to ambient temperature. However, the proposed model is entirely based on changes in detector temperature, which are not necessarily directly correlated with ambient temperature. We have verified the correlation between ambient temperature and detector temperature over a wide range of temperatures, as depicted in Figs. 5 and 6. A very strong correlation between both was shown in the entire scanner when temperature was increased, with a detector temperature change of approximately $1.4^{\circ} \mathrm{C}$ per ambient ${ }^{\circ} \mathrm{C}$. When cooling down, the correlation was still high at the bottom of the scanner, but lower in the detectors at the top of the scanner. Detector temperature also only reduces around $0.7^{\circ} \mathrm{C}$ per ambient ${ }^{\circ} \mathrm{C}$ at the top, versus approximately $1^{\circ}$ at the bottom. Detector temperature at the top of the scanner is on average also $15^{\circ} \mathrm{C}$ higher. This can be explained by the cooling system of the scanner, which functions by drawing ambient air from an inlet at the bottom and blows the air upwards to the outlet at the top of the system. During the cooling down phase, the ambient air that is drawn from the bottom cools the bottom detectors more efficiently than the top detectors. Nevertheless, after a period of stabilization of ambient temperature detector temperature at the bottom as well as at the top also stabilizes to a value which is strongly correlated with ambient temperature. Therefore, we believe that ambient temperature can be used as a surrogate measurement for detector temperature.

By acquiring the energy histogram of a ${ }^{22} \mathrm{Na}$ point source with the LabPET scanner at two different temperatures, we demonstrated that the energy histogram is indeed compressed at higher temperature. The results indicate a change in sensitivity of the detectors used in the LabPET system of approximately $6 \%$ per ${ }^{\circ} \mathrm{C}$ ambient temperature change. As described above, detector temperature changes approximately $1.4{ }^{\circ} \mathrm{C}$ per ${ }^{\circ} \mathrm{C}$ ambient temperature change. This means that $6 \%$ per ambient ${ }^{\circ} \mathrm{C}$ corresponds to $4.4 \%$ per ${ }^{\circ} \mathrm{C}$ temperature coefficient at detector level. This is comparable to what was observed by Spanoudaki et al. for an LSO-APD detector. ${ }^{13}$ In the LabPET system, LGSO and LYSO crystals are used, and a difference in temperature coefficient between detectors with these crystals was observed: $5.2 \%$ versus $3.7 \%$ per ${ }^{\circ} \mathrm{C}$ temperature change for LGSO and LYSO, respectively. This difference can be due to a difference in temperature sensitivity of both scintillator materials or could also be caused by influence of temperature on the discrimination between LYSO and LGSO detections.

As expected from the simulations, there was a strong experimental correlation between temperature and sensitivity. This was the case for immediate, short term temperature variations as well as for long term (stabilized) temperature changes. For short term temperature variations, a sensitivity change of $2.32 \mathrm{kcps} / \mathrm{MBq}$ per ambient ${ }^{\circ} \mathrm{C}$ was found, a relative decrease of sensitivity of over $10 \%$ per ${ }^{\circ} \mathrm{C}$. If care is not taken to ensure stability of the ambient temperature during long experiments, significant changes in sensitivity can therefore occur even during the experiment. In the case of dynamic experiments where images are reconstructed in different time frames, this may lead to artificial differences (or similarities) between frames, which are only caused by the scanner sensitivity variation induced by temperature instability and have no physiological meaning. For stabilized temperature changes a change in sensitivity of $-1.92 \mathrm{kcps} / \mathrm{MBq}$ per ambient ${ }^{\circ} \mathrm{C}$ was found. This is also a considerable effect and again shows the necessity of maintaining a stable scanner room temperature to allow reproducible experiments. The difference with the effect of short term variations is probably due to partial compensation of ambient temperature changes by the scanner cooling system, which lags 5 to 10 min behind ambient temperature variations. The results show good similarity with the curve obtained by simulating a detector sensitivity change of between 4 and $6 \%$ per ambient ${ }^{\circ} \mathrm{C}$. This agrees with the measurements of the energy spectrum at different temperatures. The axial sensitivity profile at $21^{\circ} \mathrm{C}$ and $24^{\circ} \mathrm{C}$ shows that sensitivity is higher at $21^{\circ} \mathrm{C}$ at each point along the axial profile. The effect of temperature on sensitivity is therefore comparable throughout the FOV, which is what would be expected with the proposed model.

The spatial resolution measurements indicate no large difference between tangential or radial resolutions acquired at different temperatures. The FWHM of the axial resolution strongly increases at increasing radial distance due to the single slice rebinning technique used on the LabPET system: from $3 \mathrm{~mm}$ FWHM at $5 \mathrm{~mm}$ radial distance to $8.8 \mathrm{~mm}$ and $10.3 \mathrm{~mm}$ at $25 \mathrm{~mm}$ radial distance at $21^{\circ} \mathrm{C}$ and $24^{\circ} \mathrm{C}$, respectively. The larger increase at $24^{\circ} \mathrm{C}$ than at $21^{\circ} \mathrm{C}$ can be attributed to the difference in temperature effect on LYSO and LGSO crystals, which are alternated axially. The FWTM of the tangential and radial resolution are slightly $(\approx 0.5 \mathrm{~mm})$ smaller at $24^{\circ} \mathrm{C}$, which is related to a reduction in scatter, as discussed below. The spatial resolution results presented here are different from those presented by Bergeron et al. ${ }^{18}$ This can be attributed to the use of single slice rebinning of data sampled from all ring differences.

Although the measurements of sensitivity and resolution using a ${ }^{22} \mathrm{Na}$ point source represent a good evaluation of basic system properties, they do not give a realistic representation of the resolution and sensitivity obtained in a real experimental situation where an animal is scanned. The main difference in such a situation is that the animal acts as a scattering medium. Therefore, a higher fraction of detected photons will be scattered photons in such a situation. A simulation of the NEMA rat-size phantom shows that the fraction of scattered photons is relatively constant in the high and low energy ranges, but changes significantly over the energy range between $470 \mathrm{keV}$ and $360 \mathrm{keV}$. When the energy histogram is compressed along the energy axis with increasing temperature, the actual lower limit that is applied will move from $250 \mathrm{keV}$ upwards and hence a smaller portion of the energy 
range with a higher scatter fraction will be included. Therefore, it is expected that the scatter fraction at higher temperatures is lower. This was observed when evaluating the true, scattered, and random count rates based on the NEMA protocol. When looking at high count rates, better results are obtained for $24^{\circ} \mathrm{C}$ than for $21^{\circ} \mathrm{C}$. The peak true count rate at $24^{\circ} \mathrm{C}$ is higher than at $21^{\circ} \mathrm{C}$, as well as the peak NEC rate. These results show that count rate effects appear at higher activity at $24^{\circ} \mathrm{C}$. This is explained by the lower sensitivity of the individual detectors at that temperature and the fact that system count rate limits apply to the total count rate. This is illustrated by the equal peak total count rate at both temperatures. As the relative fraction of true counts is higher at $24^{\circ} \mathrm{C}$, and the same peak total count rate is obtained, a higher peak true count rate and NEC rate are obtained at $24^{\circ} \mathrm{C}$.

Remarkably, the difference in total count rates between both temperatures at low phantom activity seem smaller than expected. This may be explained by the very high count rates at the start of the acquisition, which may have led to an increase in power dissipated by the detector electronics which was different at both temperatures. To eliminate these temperature loading effects, two experiments with varying temperature and a fixed low count rate were performed using a ${ }^{68} \mathrm{Ge}$ source. These experiments show that the scatter fraction is indeed lower at higher temperatures, but the true and noise equivalent count rate is also strongly reduced. One should therefore not conclude from these experiments that image quality is better at higher temperatures, as a large increase in noise at higher temperatures will also strongly affect image quality. Instead, scatter should be reduced by acquiring at low temperature and adjusting the energy window if needed. For each experiment there exists an ideal lower energy limit which will result in the highest image quality. This ideal limit depends on system properties, mainly energy resolution and geometry, as well as on the properties of the object under study. For the LabPET system investigated in this paper, the ideal lower limit will be higher when imaging a rat than when a mouse is used.

The results obtained from acquisitions with the NEMA image quality phantom also demonstrate that a higher scatter fraction is seen at $21^{\circ} \mathrm{C}$, leading to a higher spill-over-ratio. The lower recovery coefficients at $21^{\circ} \mathrm{C}$ are most likely due to the higher scatter content of the uniform section, which is used as a reference for the calculation of these coefficients. Uniformity is worse at $24^{\circ} \mathrm{C}$, which can also be observed in the slices depicted in Fig. 13, showing especially nonuniformity in the axial direction. This artifact is mainly due to the normalization, as nonuniformity is also higher when the images acquired at $21^{\circ} \mathrm{C}$ are reconstructed using a normalization scan acquired at $24^{\circ} \mathrm{C}$. It is explained by the difference in temperature sensitivity of LYSO and LGSO crystals. There is also a small contribution of higher noise at $24^{\circ} \mathrm{C}$, in the normalization scan as well as in the phantom acquisition.

The results presented in this paper also illustrate that caution should be used when interpreting system characteristics such as sensitivity and resolution, even when measured by a reproducible standard such as the NEMA NU 4-2008. Small animal PET scanners usually have a limited energy resolution, due to the small size of the scintillation crystals. By using a broad energy window it is still possible to contain approximately the entire $511 \mathrm{keV}$ photopeak within the energy window. Therefore only very few detected photons are rejected based on the energy window and a high sensitivity is obtained when measuring a point source. However, from the rat-size phantom experiment it is clear that this broad energy window also leads to a relatively high scatter fraction in a realistic imaging situation. This may lead to degradation of image quality in real experiments.

\section{CONCLUSION}

We have presented a thorough evaluation of the temperature sensitivity of the LabPET, an APD-based small animal PET scanner. The theoretical considerations presented here can also be applied to other APD-based PET systems (and future SiPM-based PET systems), although the effects may be quantitatively different. We propose that the temperature dependence of APD-based PET systems is mainly due to the temperature dependence of the gain of the APDs and the light yield of the scintillation crystal. The decrease of APD gain and light yield at higher temperatures leads to a scaling of the actual energy histogram acquired with the PET scanner along the energy axis relative to the energy histogram used for calibration. At higher temperature, this leads to a decrease of system sensitivity if the same energy window is applied as at the reference temperature. These results indicate that it is important to assure stable room temperature to obtain comparable PET images. Evaluation of the scatter fraction and count rate effects when imaging a rat-size phantom showed higher peak true count and NEC rates at higher temperature. This is due to the composition of the energy histogram at different energies. However, experiments at more realistic count rates show that the sensitivity loss is also substantial. Therefore, acquisitions should be performed at stable low temperature, the scanner should be accurately calibrated, and the lower limit of the energy histogram should be adjusted depending on the object that is being imaged.

\section{ACKNOWLEDGMENTS}

This research was supported by the European Union FP7 project SUBLIMA. Roel Van Holen is supported by the Research Foundation - Flanders, Belgium (FWO). Christian Vanhove is supported by the GROUP-ID consortium of Ghent University. The authors would like to thank Nick Van Laeken of the Department of Radiopharmacy at Ghent University for the synthesis of ${ }^{11} \mathrm{C}$. The authors declare that they have no conflict of interest.

\footnotetext{
a) Author to whom correspondence should be addressed. Electronic mail: Vincent.Keereman@ugent.be; Telephone: +32 9332 4326; Fax: +32 93324159 .

${ }^{1}$ J. L. Humm, A. Rosenfeld, and A. D. Guerra, "From PET detectors to PET scanners,” Eur. J. Nucl. Med. Mol. Imaging 30, 1574-1597 (2003).
} 
${ }^{2}$ R. Lecomte, J. Cadorette, P. Richard, S. Rodrigue, and D. Rouleau, "Design and engineering aspects of a high resolution positron tomograph for small animal imaging," IEEE Trans. Nucl. Sci. 41, 1446-1452 (1994).

${ }^{3} \mathrm{R}$. Chen et al., "Readout of scintillator light with avalanche photodiodes for positron emission tomography," Nucl. Instrum. Methods Phys. Res. A 433, 637-645 (1999).

${ }^{4}$ R. Grazioso, R. Ladebeck, and M. Schmand, "APD-based PET for combined MR-PET imaging," Proc. Intl. Soc. Mag. Reson. Med. 13, 408 (2005).

${ }^{5}$ B. J. Pichler, M. S. Judenhofer, and C. Catana, "Performance test of an LSO-APD detector in a 7-T MRI scanner for simultaneous PET/MRI," J. Nucl. Med. 47, 639-647 (2006).

${ }^{6}$ C. Catana, Y. Wu, M. Judenhofer, J. Qi, B. Pichler, and S. Cherry, "Simultaneous acquisition of multislice PET and MR images: Initial results with a MR-compatible PET scanner," J. Nucl. Med. 47, 1968-1976 (2006).

${ }^{7}$ S. I. Ziegler et al., "A prototype high-resolution animal positron tomograph with avalanche photodiode arrays and LSO crystals," Eur. J. Nucl. Med. 28, 136-143 (2001).

${ }^{8}$ J. P. Pansart, "Avalanche photodiodes for particle detection," Nucl. Instrum. Methods Phys. Res. A 387(12), 186-193 (1997).

${ }^{9}$ J. Conradi, "Temperature effects in silicon avalanche diodes," Solid-State Electron. 17, 99-106 (1974).

${ }^{10}$ H. Ando, H. Kanbe, T. Kimura, T. Yamaoka, and T. Kaneda, "Characteristics of germanium avalanche photodiodes in the wavelength region of 1-1.6 $\mu \mathrm{m}$, , IEEE J. Quantum Electron. 14, 804-809 (1978).

${ }^{11}$ J. Naud, T. Tombrello, C. Melcher, and J. Schweitzer, "The role of cerium sites in the scintillation mechanism of LSO," IEEE Nucl. Sci. Conf. R. 1, 367-371 (1995)
${ }^{12}$ S. Weber, D. Christ, M. Kurzeja, R. Engels, G. Kemmerling, and H. Halling, "Comparison of LuYAP, LSO, and BGO as scintillators for high resolution PET detectors," IEEE Trans. Nucl. Sci. 50, 1370-1372 (2003).

${ }^{13}$ V. Spanoudaki, D. McElroy, I. Torres-Espallardo, and S. Ziegler, "Effect of temperature on the performance of proportional APD-based modules for gamma ray detection in positron emission tomography," IEEE Trans. Nucl. Sci. 55, 469-480 (2008).

${ }^{14} \mathrm{M}$. Streun et al., "Pulse shape discrimination of LSO and LuYAP scintillators for depth of interaction detection in PET," IEEE Trans. Nucl. Sci. 50, 344-347 (2003).

${ }^{15} \mathrm{R}$. Fontaine et al., "The hardware and signal processing architecture of LabPET $^{\mathrm{TM}}$, a small animal APD-based digital PET scanner,” IEEE Trans. Nucl. Sci. 56, 3-9 (2009).

${ }^{16}$ M. Tetrault et al., "System architecture of the LabPET ${ }^{\mathrm{TM}}$ small animal PET scanner," IEEE Trans. Nucl. Sci. 55(5), 2546-2550 (2008).

${ }^{17}$ A. L. Goertzen et al., "NEMA NU 4-2008 comparison of preclinical PET imaging systems," J. Nucl. Med. 53, 1300-1309 (2012).

${ }^{18}$ M. Bergeron et al., "Performance evaluation of the LabPET APD-based digital PET scanner," IEEE Trans. Nucl. Sci. 56, 10-16 (2009).

${ }^{19}$ National Electrical Manufacturers Association, NEMA Standards Publication NU 4-2008: Performance Measurements of Small Animal Positron Emission Tomographs (National Electrical Manufacturers Association, Rosslyn, VA, 2008)

${ }^{20}$ S. Rechka, R. Fontaine, M. Rafecas, and R. Lecomte, "Development and validation of a GATE simulation model for the LabPET scanner," IEEE Trans. Nucl. Sci. 56, 3672-3679 (2009).

${ }^{21}$ S. Jan et al., "GATE: A simulation toolkit for PET and SPECT," Phys. Med. Biol. 49, 4543-4561 (2004). 